\title{
Preparation and Characterization of Thin Conductive Nanocomposite Film from Dispersed Multiwall Carbon Nanotubes Reinforced Chitosan/Polyvinyl Alcohol Blend
}

\author{
Fatema Tuz Zohora ${ }^{1}$, Md. Sazedul Islam ${ }^{1, *}$, Muhammad Shahriar Bashar², Papia Haque', \\ Mohammed Mizanur Rahman ${ }^{1}$ \\ ${ }^{1}$ Department of Applied Chemistry and Chemical Engineering, University of Dhaka, Dhaka, Bangladesh \\ ${ }^{2}$ Institute of Fuel Research \& Development (IFRD), Bangladesh Council of Scientific and Industrial Research (BCSIR), Dhaka, Bangladesh
}

Email address:

sazid.acce@du.ac.bd (Md. S. Islam)

${ }^{*}$ Corresponding author

\section{To cite this article:}

Fatema Tuz Zohora, Md. Sazedul Islam, Muhammad Shahriar Bashar, Papia Haque, Mohammed Mizanur Rahman. Preparation and Characterization of Thin Conductive Nanocomposite Film from Dispersed Multiwall Carbon Nanotubes Reinforced Chitosan/Polyvinyl Alcohol Blend. Science Research. Vol. 7, No. 6, 2019, pp. 78-84. doi: 10.11648/j.sr.20190706.12

Received: May 27, 2019; Accepted: October 26, 2019; Published: November 5, 2019

\begin{abstract}
In this study, surfactant dispersed MWCNTs were introduced as nanofillers into poly (vinyl) alcohol (PVA) and Chitosan (Cs) blend (ratio 50:50 wt \%, optimized) by solution casting method to fabricate PVA/Cs/MWCNTs nanocomposite films. These nanocomposites were subjected to different characterization to study the variation of properties with different amount of MWCNTs loading. Various techniques, such as Optical microscopy (OM), Fourier transform infrared spectroscopy (FTIR), thermogravimetric analysis (TGA, DTGA), differential scanning calorimetry (DSC), impedance analyzer (IA), scanning electron microscopy (SEM) and universal testing machine (UTM) were used to study the physicochemical, morphological, electrical and thermo-mechanical properties of the nanocomposite films. The experimental results of FTIR illustrated that strong interaction among MWCNTs, Cs and PVA facilitated the crystallization of PVA and prevented the agglomeration of MWCNTs in the composite film. Tensile strength of the nanocomposite containing $1 \mathrm{wt} \%$ MWCNTs increased by $61.51 \%$ and elongation at break decreased by $20.07 \%$ in comparison to that of pure PVA/Cs blend film. Similarly, the conductivity of the nanocomposite containing $1 \mathrm{wt} \%$ MWCNTs was highest at $40 \mathrm{~V}$ with the value of $1.99 \times 10^{-3} \mathrm{~S} / \mathrm{cm}$.
\end{abstract}

Keywords: Chitosan, Blends, Nanocomposites, Thermal Properties, Mechanical Properties

\section{Introduction}

Nanobiocomposites are currently one of the most studied topics in environmentally friendly polymer research. Synthetic polymers used in nanocomposites have already being considered as a major threat of waste after use [1-3]. A large number of publications have reflected the increasing interest on polymers modification by polymers grafting or crosslinking and even with blending of different polymers to obtain composites with improved properties $[4,5]$. Blends of natural polymer (chitosan, starch, gelatin, collagen, cellulose etc) and synthetic polymer (PVA, PGA, PLA, polystyrene etc.) are intensively investigated to improve biodegradability of them and to prepare composites with various applications, like electrical, biomedical, food industry, etc [6].
In order to fabricate nanocomposites, inorganic materials are used as nanofillers to improve the properties of polymers $[7,8]$. Carbon nanotubes (CNTs) can be used as filler due to their nano size, high aspect ratio and most importantly, their high mechanical strength, electrical and thermal conductivity $[9,10]$. However, CNTs are hydrophobic in nature due to lack of functional groups on its surface, thus tends to agglomerate as strong van der Waal's force exist among nanotubes. Such interactions restrict the dispersion of CNTs in polymer matrix and limit the application of CNTs [11]. To overcome this drawback of CNTs, non-covalent modification of CNTs with surfactant and biopolymers have acquired attention of researchers. Surfactants get adsorbed on the surface of nanotubes, reduce agglomeration between themselves and act as adhesion promoters, leading to the 
formation of highly adhesive interfaces between individual nanotubes and polymeric matrix. Triton X100 (nonionic surfactant) with long aromatic hydrocarbon chain separates the CNTs more effectively than the one with short chain [12]. Among all types of CNTs, multiwalled carbonanotubes (MWCNTs) are acknowledged as ideal reinforcing fillers for biopolymers due to its high aspect ratio (upto $132,000,000: 1)$, specific area $\left(10^{3} \mathrm{~m}^{2}\right)$, and tensile strength (more than $200 \mathrm{GPa}$ ) etc. [13].

Chitosan is a naturally abundant, low cost polysaccharide which is mainly composed of $\beta$-(1,4)-linked 2-deoxy-2amino-D-glucopyranose units, is the deacetylated product of chitin, poly (N-acetyl-D-glucosamine). It is one of the most important polymer in composites preparation among naturally derived polymers, due to its biodegradability, biocompatibility, bioadhesivity and responsiveness [14]. Chitosan can be used to ensure efficient load transfer among dispersed MWCNTs as polymer matrix in composites. When chitosan is used as a polymer matrix in nanocomposites, it imparts strength along with significant amount of brittleness. To overcome this drawback of chitosan, another polymer with enough flexibility can be blended with chitosan. PVA is a non-toxic, water soluble, biocompatible and biodegradable polymer with very good flexibility.

In this study, PVA solution in water $(5 \mathrm{wt} \%) /$ Chitosan in acetic acid solution (2 wt.\%) mixture with the ratio of 50:50 (w/w, ) was used to fabricate nanocomposites with different MWCNTs loading and subjected to mechanical, thermal and electrical characterizations.

\section{Experimental}

\subsection{Materials}

Pristine MWCNTs were supplied by Sigma Aldrich Company, USA (Diameter: 6 to $9 \mathrm{~nm}(\geq 95 \%)$, Length: $5 \mu \mathrm{m}$ ( $\geq 95 \%$ ), Melting Point: $3652-3697^{\circ} \mathrm{C}$, Density: $2.1 \mathrm{~g} / \mathrm{ml}$ (approx.) at $\left.25^{\circ} \mathrm{C}\right)$. Polyvinyl alcohol (PVA) $\left(\mathrm{M}_{\mathrm{w}} 89,000\right.$ 98,000, >99\%) was supplied by Sigma-Aldrich, UK. Chitosan with deacetylation degree of $79.4 \%$ (calculated using the baseline method of Domszy et al. [15] produced from locally collected waste prawn shell, following the process described in our previous investigation [16]. Triton X100 (T-Octylphenoxyethoxy ethanol) was purchased from Sigma Chemical Company, USA. SDS (sodium dodecyl sulfate) $(>98.5 \%)$ was purchased from Sigma-Aldrich, UK. Acetic acid (100\%), and hydrochloric acid (37\%) were obtained from Merck, Germany.

\subsection{Methods}

\subsubsection{Preparation of MWCNTs Solution}

MWCNTs $(50 \mathrm{mg})$ was added into distilled water $(100 \mathrm{ml})$ containing $2 \mathrm{wt} \%$ surfactant (Triton X100 or SDS). The solution was then ultrasonicated for 72 hours in $200 \mathrm{KHz} 50$ $60 \mathrm{~W}$ bath ultrasonicator. The solution was centrifuged at $10000 \mathrm{rpm}$ in a High Speed Refrigerated Centrifuge (H-9R Kokusan Corporation, Japan) for 1 hour followed by filtration of the solution to remove the non-dispersed agglomerated MWCNTs.

\subsubsection{Preparation of the Nanocomposite Film}

Surfactant dispersed MWCNTs aqueous solution $(0.25 \%$, $0.5 \%, 0.75 \%$ and $1 \%$ in $\mathrm{w} / \mathrm{w}$ basis) was added into a polymeric blend of 5\% PVA and 2\% Chitosan (ratio 50:50, $\mathrm{wt} \%$ ) and stirred for 45 minutes (at $40-45^{\circ} \mathrm{C}$ ) to homogenize and sonicated again for 1 hour to remove air bubbles. The PVA/Chitosan/MWCNTs mixtures were refrigerated for 10 minutes in a deep refrigerator and poured into silicon cloth covered flat glass plate $(6 \mathrm{~cm} \times 10 \mathrm{~cm} \times 1 \mathrm{~cm})$ for film formulation. Then the glass plates were kept in a laminar flow (niive LN-12) for 25 to 34 hours. Thin films with average thickness of $0.30 \mathrm{~mm}$ were peeled off the glass plate and kept in the desiccator for further use.

\subsection{Characterization}

\subsubsection{Trinocular Microscopy}

The optical images of the dispersion of MWCNTs in distilled water in the presence of SDS and Triton X100 were observed using CKX41 Inverted Microscope (Olympus, USA).

\subsubsection{FTIR Spectroscopy}

Fourier transform infrared (FT-IR) spectra of the samples were obtained by using a FT-IR spectrophotometer (Model 8400 S, Shimadzu Corporation, Japan) in the range of 4000 $400 \mathrm{~cm}^{-1}$, resolution: $4 \mathrm{~cm}^{-1}$; no. of scans: 30 times.

\subsubsection{Mechanical Properties}

The tensile strength (TS) and elongation at break (EB\%) of the films were determined by Universal Testing Machine (UTM) of "Thumbler Z3 Series" testing machine, Germany. The rectangle shaped test specimens were cut according to ISO-527-3 with length $100 \mathrm{~mm}$ and width $10 \mathrm{~mm}$. The load range was $500 \mathrm{~N}$ and the initial distance of separation was 65 $\mathrm{mm}$ and the tests were performed at room temperature at velocity $10 \mathrm{~mm} / \mathrm{sec}$.

\subsubsection{Morphological Analysis}

The morphology of prepared PVA/Chitosan blend film and nanocomposites were investigated using JEOL JSM- 6490LA scanning electron misroscope (JEOL Ltd., Japan) with 1000$2500 \mathrm{X}$ magnification and an accelerating voltage of 20-25 $\mathrm{kV}$. The SEM specimens were coated with platinum.

\subsubsection{Thermal Analysis}

To characterize the decomposition and thermalstability of nanocomposites with different MWCNTs loading were observed using a thermogravimeric analyzer (TGA-50H Shimadzu, Japan) from room temperature to $600^{\circ} \mathrm{C}$ with a heating range of $10^{\circ} \mathrm{C} / \mathrm{min}$, under nitrogen atmosphere with a flow rate of $10 \mathrm{ml} / \mathrm{min}$. Differential scanning calorimeter (DSC-60H, Shimadzu, Japan) was used to examine phase transition and degradation properties of the nanocomposites as a function of temperature. The samples were scanned within the temperature range from room temperature to 
$400^{\circ} \mathrm{C}$ with a heating rate of $10^{\circ} \mathrm{C} / \mathrm{min}$, under nitrogen atmosphere with a flow rate of $20 \mathrm{ml} / \mathrm{min}$.

\subsubsection{Conductivity Measurements}

The conductivity of the PVA/Chitosan blend film and PVA/Chitosan/MWCNTs nanocomposites were measured directly using "Four Probe Impedance Analyzer"(Keithlink, Korea). The thickness of the nanocomposite films were measured using screw gauge.

\section{Results and Discussion}

To solve the aggregation problem of MWCNTs in solution, SDS and Triton X100 were used separately as surfactant. Triton $\mathrm{X} 100$ gave better dispersion in the solvent (dist. water) than SDS (Figure 1). Due to the presence of benzene ring in Triton X100 structure, it shows strong interaction with the nanotube surface compared to SDS. $\pi$ - like stacking of the benzene rings onto the surface of CNTs is believed to increase the bindings and surface coverage of surfactant molecules to CNTs [17]. The photographs in Figure 1 clearly showed sedimentation for using SDS in the dispersion of MWCNTs.

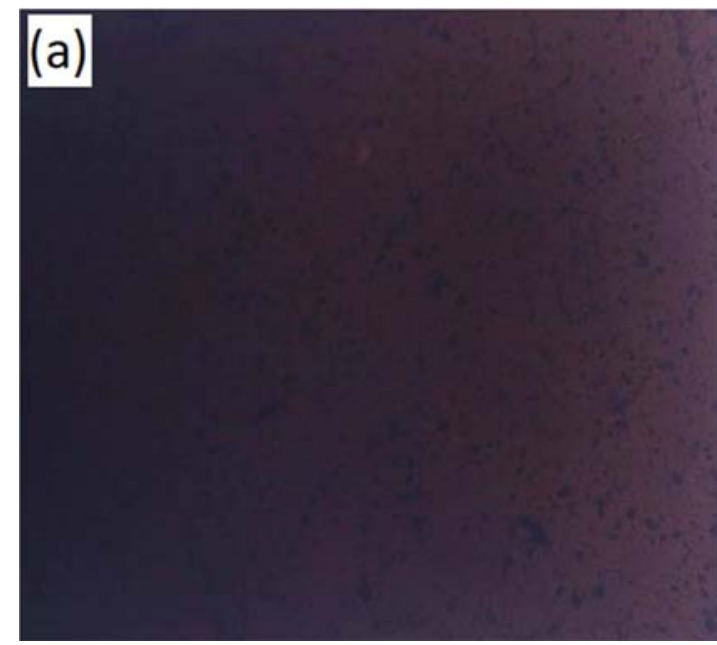

Figure 2. Optical microscopic images of MWCNTs dispersion a) in Triton X100 and b) in SDS.

\subsection{FTIR Spectroscopy}

The FTIR spectra of PVA (5\%)/Chitosan (2\%) (50:50, wt $\%$ ) blend film and PVA/Chitosan/MWCNTs nanocomposites at different ratios are presented in Figure 3. For PVA/Chitosan blend film, two distinct peak at $1408 \mathrm{~cm}^{-1}$ and $1548-1560 \mathrm{~cm}^{-1}$ are observed. The peak at $1408 \mathrm{~cm}^{-1}$ confirms the presence of $-\mathrm{COOH}$ groups in the polymer. The peak at $1548 \mathrm{~cm}^{-1}$ is due to the symmetric deformation of $\mathrm{NH}_{3}$ resulting from ionization of primary amino group in the acidic medium. The peak at $1700 \mathrm{~cm}^{-1}$ is characteristic peak of carboxylic acid dimers which are formed from acetic acid solution in which chitosan was dissolved. Those peaks between $1210-1300 \mathrm{~cm}-1$ is due to $-\mathrm{C}=\mathrm{H}$ vibration. Two peaks at $3340 \mathrm{~cm}^{-1}$ and $1422 \mathrm{~cm}^{-1}$ confirms the presence of $\mathrm{OH}$ stretching and bending. High intermolecular interaction of H-bonding between functional groups of polymer enhance

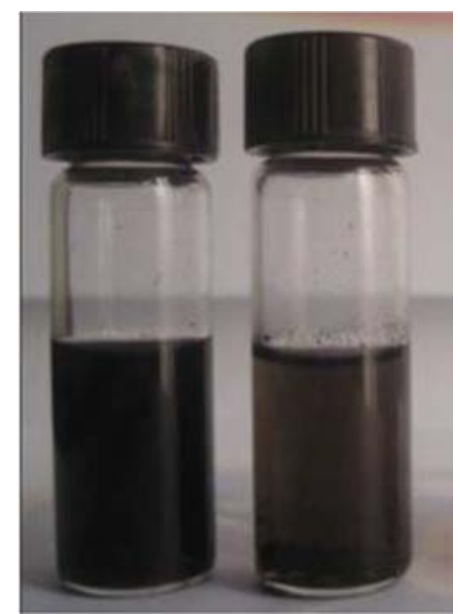

Figure 1. Photograph of dispersed MWCNTs in a) 2\% Triton X100 solution and b) $2 \%$ SDS solution (after 7 days).

Similarly, optical microscopic images (Figure 2) of the dispersed MWCNTs solution also confirmed that Triton $\mathrm{X} 100$ was better dispersant than SDS. As the dispersion of MWCNTs were better in Triton X100, so this surfactant was used to disperse MWCNTs in the final composite.

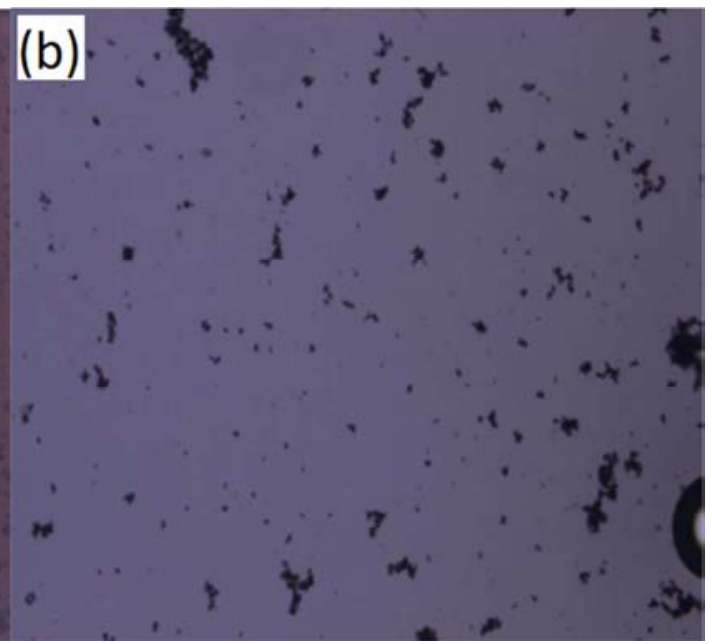

physical stability. This H-bonding cause prevention of crystallization of chitosan in the polymer matrix but don't hamper the PVA crystallinity.

It is clear from the FTIR spectrum of nanotubes loaded PVA/Chitosan film that the addition of nanotube does not causes any characteristic (except one) new bond because the bonding frequencies related to $-\mathrm{OH}, \mathrm{C}-\mathrm{O}$ or $-\mathrm{NH}$ of the PVA/Chitosan matrix which have not been changed. But there is a characteristic absorption peak at $1643 \mathrm{~cm}^{-1}$ is ascribed to the amide $\mathrm{I}\left(\mathrm{C}=\mathrm{O}\right.$ stretching of $\left.-\mathrm{NHCOCH}_{3}\right)$ as shown in the spectrum of PVA/Chitosan and MWCNTs blend film, which shifts $7 \mathrm{~cm}^{-1}$ (from $1655 \mathrm{~cm}^{-1}$ ) compared with that of PVA/Chitosan film. It indicates that the strong interaction between the hydrophobic $-\mathrm{COCH}_{3}$ of chitosan and the surface of MWCNTs, and thus prevents effective agglomeration of MWCNTs [18, 19]. 


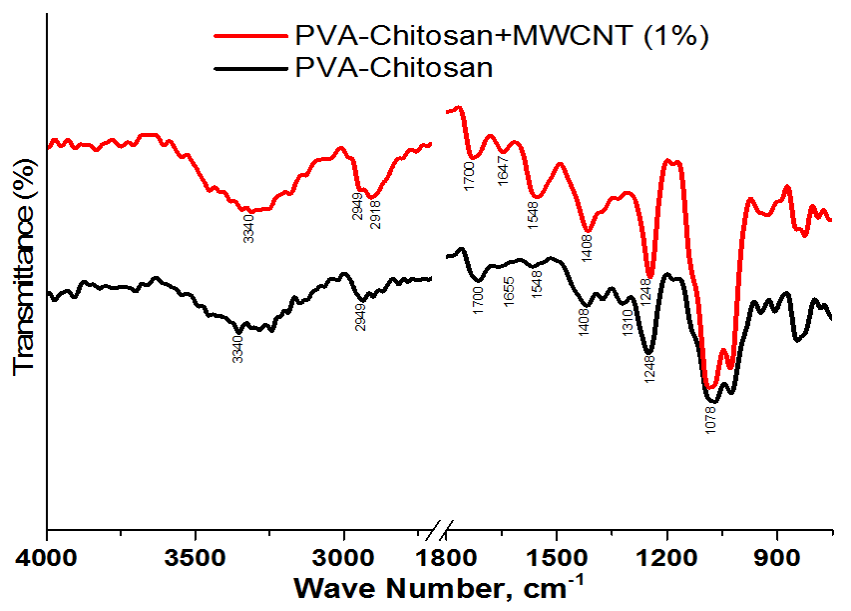

Figure 3. FTIR spectrum of PVA/Chitosan blend and PVA/Chitosan /MWCNTs (1 wt\%) nanocomposite.

\subsection{Mechanical Properties}

The effect of MWCNTs loading on the mechanical properties of nanocomposites are significantly higher than those of pure PVA/Chitosan (PC) blend film. From figure 4 it is observed that, TS has increased sharply with an increase in MWCNTs loading from 0.25 to $1 \mathrm{wt} \%$ and elongation at break also increased gradually with an increase in MWCNTs loading. TS value is highest for $1 \mathrm{wt} \%$ MWCNTs loading because fillers (PVA and chitosan) take a significant larger share of the load [20]. Another reason behind increasing TS is that addition of MWCNTs promoted the degree of crytallinity of PVA chains [21]. It has been established by other researcher that the presence of ordered polymer coating is the main reason leading to the enhanced mechanical strength [22]. However, with the increase of MWCNTs loading to $2 \mathrm{wt} \%$, TS is observed to decrease as this caused agglomeration of nanotubes in the matrix. With the loading of MWCNTs ( $1 \mathrm{wt} \%$ ), TS of nanocomposite increases around $61 \%$ and elongation at break decreases around 20\% (as MWCNTs loading decreased the elasticity of the film) in comparison to those of the pure PVA/Chitosan blend film.

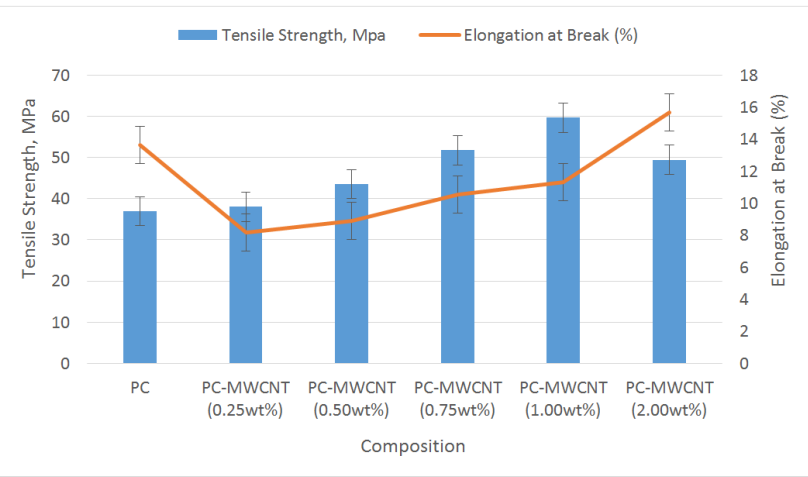

Figure 4. Tensile strength and elongation at break of PVA/Chitosan /MWCNTs nanocomposites with different MWCNTs loading.

\subsection{Morphological Analysis}

SEM images of fractured cross-section of PVA/Chitosan blend film and PVA/Chitosan /MWCNTs nanocomposites are shown in figure 5 (a) and (b) respectively. Homogenous blending of PVA and Chitosan is observed from the SEM image of PVA/Chitosan blend film. The reinforcement of MWCNTs in PVA/Chitosan blend is clearly visible in the SEM image of PVA/Chitosan /MWCNTs nanocomposites.

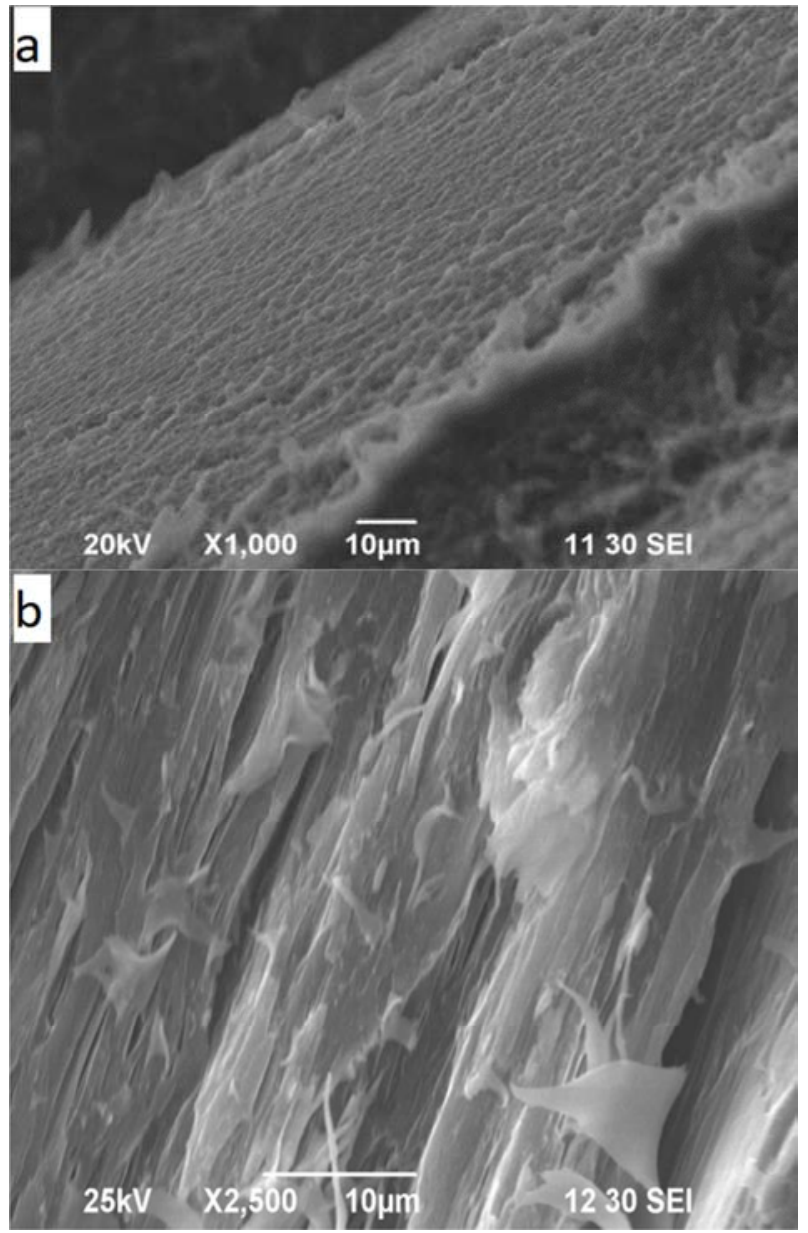

Figure 5. SEM images of a) fractured cross-section of PVA/Chitosan blend film (porous structure) and b) fractured cross-section of the PVA/Chitosan /MWCNTs nanocomposites.

\subsection{Thermal Analysis}

The thermogram of PVA/Chitosan blend film and PVA/Chitosan/MWCNTs nanocomposites is shown in Figure 6. The initial weight loss upto $150^{\circ} \mathrm{C}$ is due to removal of absorbed water from the composite. Two significant drops starting at around $250^{\circ} \mathrm{C}$ and $430^{\circ} \mathrm{C}$ are clear from the given thermogram which indicates two major thermal degradation of the composite material. The first breakdown of the curve is because of melting and the second one is for decomposition of the composite. From the TGA curves, it is observed that with the introduction of MWCNTs in PVA/Chitosan blend, thermal degradation of composite started at higher temperature. This is because the presence and good dispersion of MWCNTs which suppress the polymer chain mobility. Interaction between polymer molecules and CNT restricts the polymer shrinkage which 
leads to thermal stability of nanocomposites.

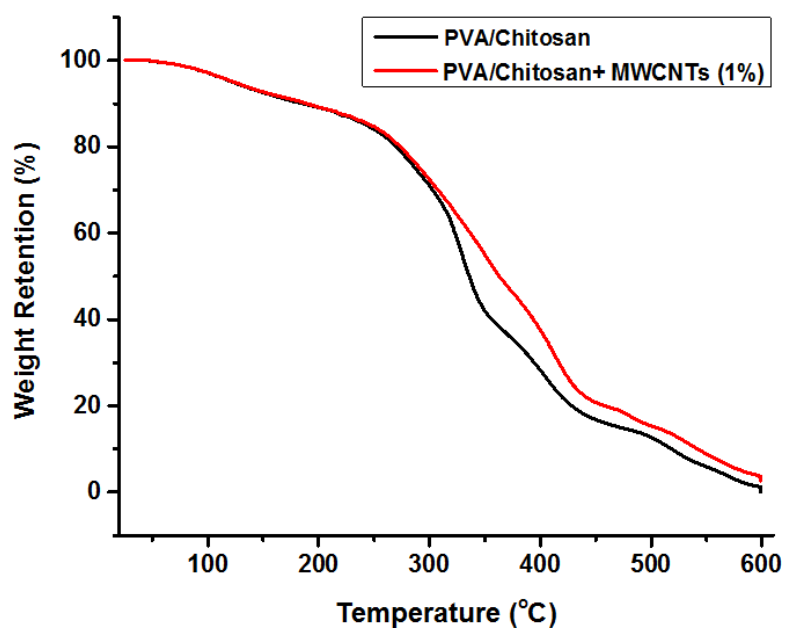

Figure 6. TGA curves of PVA/Chitosan blend film and PVA/Chitosan/MWCNTs nanocomposites.

The effect of nanotubes presence on polymer crystallization was examined under differential scanning calorimetry (DSC) by heating samples upto $400^{\circ} \mathrm{C}$. It is evident from the DSC curve that addition of MWCNTs in PVA/Chitosan blend matrix increases the thermal stability (right shift) of the nanocomposites. This is because a good degree of bonding between the PVA/Chitosan matrix and the nanotubes strongly prevents the polymer from melting at its bulk melting temperature. As a result the melting peak of the nanocomposites are found to increase $7^{\circ} \mathrm{C}\left(307^{\circ} \mathrm{C}-314^{\circ} \mathrm{C}\right)$, higher than the PVA/Chitosan blend film. The DSC thermograms of all the composites show one single broad glass transition temperature peak within the range $70^{\circ} \mathrm{C}$ $98^{\circ} \mathrm{C}$, which supports single-phase behavior of the blending system and a good degree of bonding among the polymers and nanotubes.

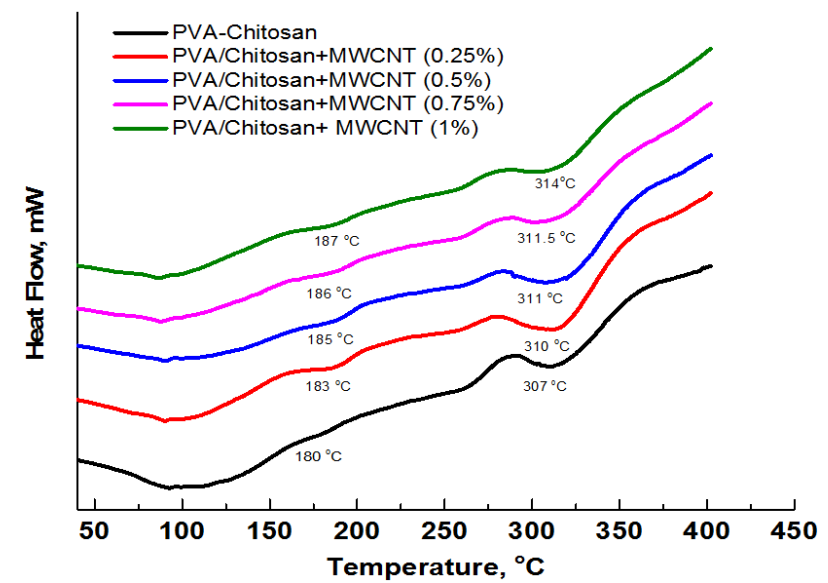

Figure 7. DSC curves of the nanocomposites with different MWCNTS loading.

\subsection{Measurement of Electrical Conductivity, Sheet Resistance and Volume Resistivity}

The result of electrical conductivity, sheet resistance and volume resistivity measurement at applied voltages of $30 \mathrm{~V}$ to $40 \mathrm{~V}$ (thickness of the film $(0.30 \pm 0.03) \mathrm{mm})$ with different MWCNTs loading is shown in Figure 8. From the conductivity measurement, it is observed that the incorporation of MWCNTs in chitosan/PVA film improves the electrical conductivity of the film. Moreover, conductivity increases with increase in applied voltage and it is found that conductivity of the nanocomposite containing 1 wt $\%$ MWCNTs is highest at $40 \mathrm{~V}$ with the value of $1.99 \times 10^{-}$

${ }^{3} \mathrm{~S} / \mathrm{cm}$. Addition of MWCNTs in polymer matrix increases the conductivity of the nanocomposites because increasing MWCNTs loading helps to reach the percolation threshold which is a three-dimensional conductive network among CNTs and thus electron conduction is facilitated through a tunneling mechanism among nanotubes. Consequently, both sheet resistance and volume resistivity of the nanocomposites decrease with increase in MWCNTs loading and applied voltage. The sheet resistance and volume resistivity is minimum at $40 \mathrm{~V}$ with the value of $13.7 \times 10^{3} \mathrm{ohms} / \mathrm{square}$ and $0.402 \times 10^{3}$ ohms-cm respectively.

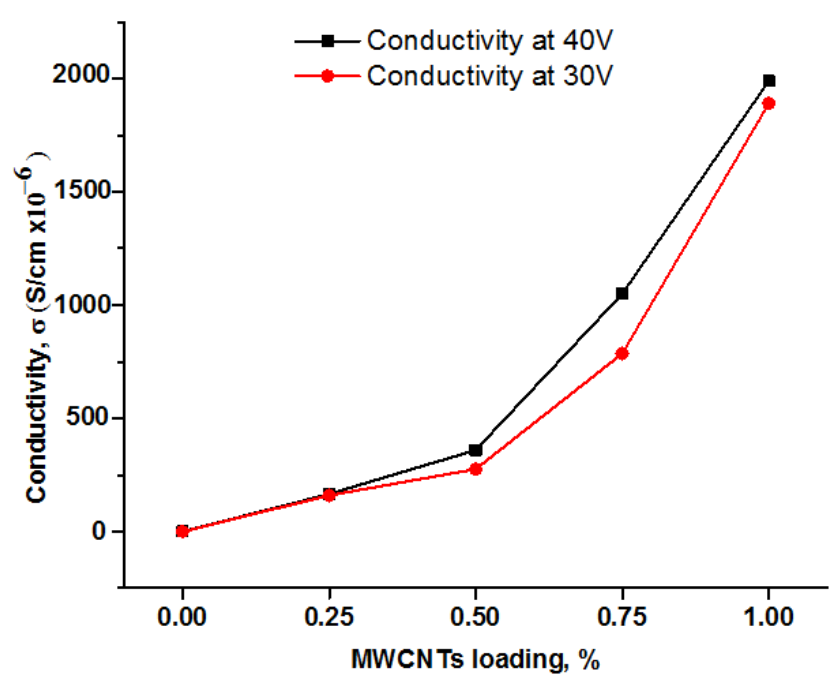

(a)

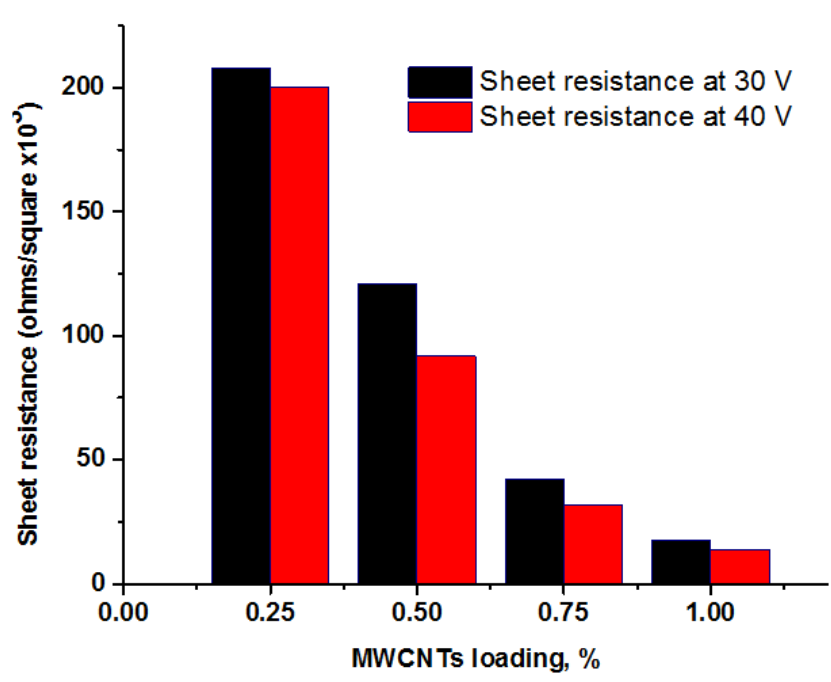

(b) 


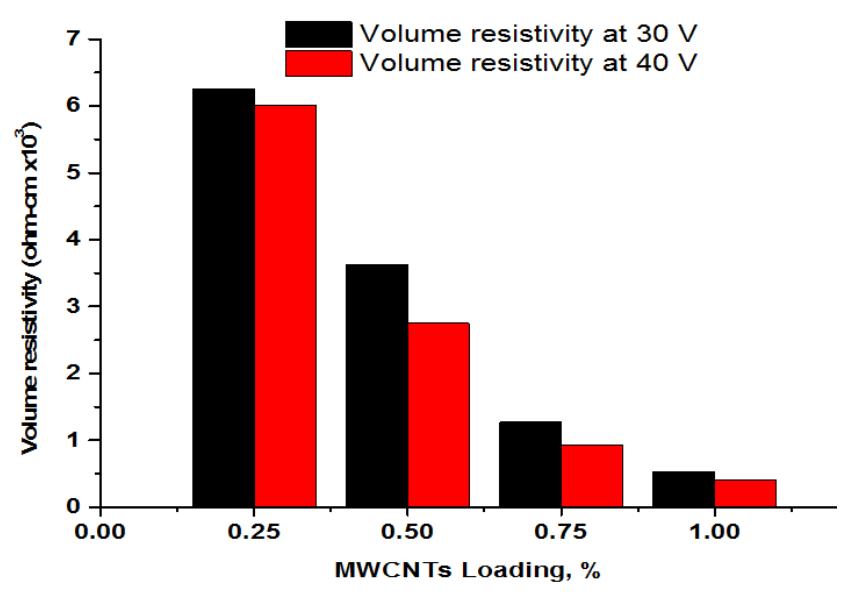

(c)

Figure 8. (a) Conductivity, (b) sheet resistance and (c) volume resistivity of nanocomposites at different MWCNTs loading.

\section{Conclusions}

In this research, MWCNTs incorporated PVA/Chitosan nanocomposite films were successfully prepared by solution casting method. The effects of dosage of different percentage of MWCNTs on PVA/Chitosan blend were investigated and improved results were obtained in comparison to neat PVA/Chitosan blend films. These characterizations prove that surfactant treated MWCNTs dispersed well in PVA/Chitosan blend matrix. Such successful incorporation of MWCNTs (upto $1 \mathrm{wt} \%$ ) in polymer matrix gave nanocomposites with better mechanical, thermal and electrical properties. Extent of compatibilization of MWCNTs with the polymer matrix in the nanocomposites were confirmed by FTIR. Incorporation of MWCNTs in chitosan/PVA film improves the electrical conductivity of the film and as a consequence both sheet resistance and volume resistivity of the nanocomposites decrease with increase in MWCNTs. The promising results obtained from different characterization tests suggest that this conductive biodegradable film has potential to be used in thermomechanical and electrical applications.

\section{Conflicts of Interest}

The authors declare they have no conflict of interest.

\section{Acknowledgements}

The authors acknowledge their affiliated institutions.

\section{References}

[1] Coelho, C., et al., Functionalisation of polybutylene succinate nanocomposites: from structure to reinforcement of UVabsorbing and mechanical properties. Rsc Advances, 2012. 2 (12): p. 5430-5438.
[2] Petersson, M. and M. Stading, Water vapour permeability and mechanical properties of mixed starch-monoglyceride films and effect of film forming conditions. Food Hydrocolloids, 2005. 19 (1): p. 123-132.

[3] $\mathrm{Wu}, \mathrm{H}$., et al., Preparation and characterization of chitosan $/ \alpha$-zirconium phosphate nanocomposite films. Polymer International, 2010. 59 (7): p. 923-930.

[4] Utracki, L., Polymeric nanocomposites: compounding and performance. Journal of nanoscience and nanotechnology, 2008. 8 (4): p. 1582-1596.

[5] Barlow, J. and D. Paul, Polymer blends and alloys - a review of selected considerations. Polymer Engineering \& Science, 1981. 21 (15): p. 985-996.

[6] Vasile, C., Environmentally degradable materials based on multicomponent polymeric systems. 2009: CRC Press.

[7] Paiva, M., et al., Mechanical and morphological characterization of polymer-carbon nanocomposites from functionalized carbon nanotubes. Carbon, 2004. 42 (14): p. 2849-2854.

[8] Bhat, A. and A. Banthia, Preparation and characterization of poly (vinyl alcohol)-modified red mud composite materials. Journal of applied polymer science, 2007. 103 (1): p. 238-243.

[9] Ajayan, P. M., et al., Single-walled carbon nanotube-polymer composites: strength and weakness. Advanced materials, 2000. 12 (10): p. 750-753.

[10] Wang, S.-F., et al., Preparation and mechanical properties of chitosan/carbon nanotubes composites. Biomacromolecules, 2005. 6 (6): p. 3067-3072.

[11] Adsul, M. G., D. A. Rey, and D. V. Gokhale, Combined strategy for the dispersion/dissolution of single walled carbon nanotubes and cellulose in water. Journal of Materials Chemistry, 2011.21 (7): p. 2054-2056.

[12] Rastogi, R., et al., Comparative study of carbon nanotube dispersion using surfactants. Journal of colloid and interface science, 2008. 328 (2): p. 421-428.

[13] Wang, X., et al., Fabrication of ultralong and electrically uniform single-walled carbon nanotubes on clean substrates. Nano letters, 2009.9 (9): p. 3137-3141.

[14] Gil, E. S. and S. M. Hudson, Stimuli-reponsive polymers and their bioconjugates. Progress in polymer science, 2004. 29 (12): p. 1173-1222.

[15] Domszy, J. G. and G. A. Roberts, Evaluation of infrared spectroscopic techniques for analysing chitosan. Die Makromolekulare Chemie: Macromolecular Chemistry and Physics, 1985. 186 (8): p. 1671-1677.

[16] Rashid, T. U., et al., A new approach for the preparation of chitosan from $\gamma$-irradiation of prawn shell: effects of radiation on the characteristics of chitosan. Polymer International, 2012. 61 (8): p. 1302-1308.

[17] Liu, J.-F. and W. A. Ducker, Self-assembled supramolecular structures of charged polymers at the graphite/liquid interface. Langmuir, 2000. 16 (7): p. 3467-3473.

[18] Chuang, W.-Y., et al., Properties of the poly (vinyl alcohol) /chitosan blend and its effect on the culture of fibroblast in vitro. Biomaterials, 1999. 20 (16): p. 1479-1487. 
[19] Srinivasa, P., et al., Properties and sorption studies of chitosan-polyvinyl alcohol blend films. Carbohydrate Polymers, 2003. 53 (4): p. 431-438.

[20] Zhang, X., et al., Poly (vinyl alcohol) /SWNT composite film. Nano letters, 2003.3 (9): p. 1285-1288.

[21] Minus, M. L., H. G. Chae, and S. Kumar, Single wall carbon nanotube templated oriented crystallization of poly (vinyl alcohol). Polymer, 2006.47 (11): p. 3705-3710.

[22] Coleman, J. N., et al., Reinforcement of polymers with carbon nanotubes. The role of an ordered polymer interfacial region. Experiment and modeling. Polymer, 2006. 47 (26): p. 85568561. 